\title{
Chronic Cerebral Ischemia-Induced Memory Impairment After Inhibition of BDNF by Interleukin-1 Signaling Pathway
}

\author{
Lan Xian-wu*, Cui Liang, Gui Wen-shan \\ Department of Cardiology, The First Affiliated Hospital of Jinan University, Guangzhou, People's Republic of China
}

Email address:

lanxianwu2017@126.com (Lan Xian-wu)

${ }^{*}$ Corresponding author

To cite this article:

Lan Xian-wu, Cui Liang, Gui Wen-shan. Chronic Cerebral Ischemia-Induced Memory Impairment After Inhibition of BDNF by Interleukin-1 Signaling Pathway. American Journal of Internal Medicine. Vol. 5, No. 4, 2017, pp. 52-56. doi: 10.11648/j.ajim.20170504.11

Received: March 28, 2017; Accepted: April 10, 2017; Published: June 15, 2017

\begin{abstract}
Objective To observe the abnormal expression of IL-1ßin hippocampus after bilateral common carotid artery ligation and to evaluate its effects on memory impairment induced by chronic cerebral ischemia. Methods Bilateral common carotid artery ligation (2-vessel occlusion, 2VO) was performed in wild type (Wild Type WT) and interleukin -1 receptor knock-out (IL-1 Resept Knock Out, IL-1R KO) C57 mice (male) to produce chronic cerebral ischemia mouse models. After the operation, their memory was detected with eight-arm radial maze test and the novel object recognition test, and the level of IL-1 $\beta$ and BDNF in hippocampus was detected with Western Blot. Results The average number of working memory errors of the KO-2VO mice was significantly decreased $(\mathrm{P}<0.05)$, and the recognition index was significantly increased $(\mathrm{P}<0.05)$ compared with those of the WT-2VO mice. Dramatic decrease in IL-1 $\beta$ in hippocampus $(\mathrm{P}<0.05)$ and substantial increase in BDNF $(\mathrm{P}<0.05)$ were observed in the KO-2 $\mathrm{VO}$ mice. In the behavioral experiment and Western Blot, there was no significant difference between the KO-sham mice and the WT-sham controls. Conclusion Increase in the level of the IL-1 $\beta$ in hippocampus after bilateral common carotid artery ligation can lead to decrease in the level of the BDNF, which may cause memory impairment.
\end{abstract}

Keywords: Chronic Cerebral Ischemia, Bilateral Common Carotid Artery Ligation, Microglia, Interleukin-1 $\beta$, Brain-Derived Neurotrophic Factor (BDNF), Working Memory Impairment

\section{Introduction}

In recent years, with the rapid development of economy and continuing improvement of life quality, the aged population has been increasing. As a result, age-associated memory disorders (including senile dementia, vascular dementia and Bins wanger disease, etc.) caused by chronic cerebral ischemia has given rise to more and more concerns. Chronic cerebral ischemia (CCI) is mainly caused by chronic cardiovascular and cerebrovascular diseases, such as hypertension, atherosclerosis, diabetes, cardiomyopathy, myocarditis, anemia, microthromboembolism, thrombocytosis and increased blood viscosity. These factors usually cause stenosis and low perfusion in brain blood vessels, resulting in long-term reduction in cerebral blood flow which ultimately destroys the normal metabolism of brain cells. As a result, brain function declines, often presenting with cognitive impairment involving memory and emotion [1]. Studies have shown that various cytokines including inflammatory cytokines, chemotactic cytokines, etc. undergo alterations under chronic cerebral ischemia, in which IL-1 $\beta$ plays an important role [2-3]. Brain-derived neurotrophic factor (BDNF), mainly expressed in the central nervous system, nourishes nerves and increases the synaptic plasticity in the neural network, and can influence learning and memory by changing hippocampuslong-term-potentiation (LTP) [4]. There are a large number of evidences that BDNF decline in hippocampus caused by chronic cerebral ischemia is a basic factor for working memory impairment [5-6] but its mechanism remains unclear. This study is to test whether interleukin-1 receptor knockout can alter the abnormal expression of BDNF to improve CCI-induced memory impairment in interleukin -1 receptor knock-out CCI mouse models produced by bilateral common carotid artery ligation. 


\section{Materials and Methods}

\subsection{Model Establishment and Experimental Design}

In this study, wild type (WT) C57 mice (adult males) and interleukin-1 receptor knock-out (IL-1 Receptor Knock Out, IL-1R KO) C57 mice (adult males) of $20 \sim 25 \mathrm{~g}$ were divided into the following four groups: sham group $(n=8)$; two-vessel occlusion (2VO) [7] group $(\mathrm{n}=8)$; IL-1R KO-sham group (n $=8)$; IL-1R KO-two-vessel occlusion $(\mathrm{KO}-2 \mathrm{VO})$ group $(\mathrm{n}=$ 8). Mice in $2 \mathrm{VO}$ group and $\mathrm{KO}-2 \mathrm{VO}$ group received fasting for solids and liquids 12 hours before operation and were anesthetized with $10 \%$ chloral hydrate at a dose of $4 \mathrm{ml} / \mathrm{kg}$. The procedure was carried out in the supine position with an incision in the midline of anterior neck. The left and right common carotid arteries were fully exposed with blunt dissection to be ligated and then the surgical incision was sutured without any damage to vagus nerves. Mice in sham group and KO-sham group were treated with the same procedure except that no ligation was conducted. After surgery, 4 groups were fed routinely in a warm condition. The narrowing of palpebral fissure and blepharoptosis when the mice wake up indicated that the ligation was successful.

The detailed experimental procedures are shown in Figure 1A. All mice were given 5 days to adapt to the cage and proper amount of food and water in a $12 \mathrm{~h} / 12 \mathrm{~h}$ day/night cycle with moderate humidity. On day $0,2 \mathrm{VO}$ and sham surgery were performed and then the mice were returned to the cage. Eight-arm radial maze test was performed on the 36th-42th day after surgery and novel object recognition test on the 43th-45th day. On the 46th day, the brains were harvested and hippocampuses were extracted for Western Blot test. All tests were double-blind.

\subsection{Novel Object Recognition Test}

Novel object recognition test was designed according to the principle that the mouse has stronger desire to learn novel objects after remembering old ones. Black round open-top box with $40 \mathrm{~cm}$ in diameter and height, black walls and bottom was used as test box. Before the test, the smell the mice left on the test boxes and test tools was cleaned. The mice received a ten-minute adaptation training a day for two days in empty boxes after which the novel object recognition test was conducted. During the novel object recognition test, two objects were placed in a box at first, then the mice for 5 minutes, leaving them to learn the objects freely. This was the cognitive stage for samples. After the cognitive stage, the object learnt less by the mice was replaced with a new object. After 10 minutes, the mice were returned to the box for another 5 minutes and their learning on both new and old objects was observed. This process was called acquired cognitive stage (short-term memory test). The test was performed under the same condition among all groups. The recognition index refers to the percentage of learning times of an object in total learning times of two objects. The smaller the recognition index is, the lower the short-term memory capacity.

\subsection{Eight-Arm Radial Maze Test}

Working memory capacity of mice was tested with eight-arm radial maze test. The eight-arm radial maze consists of eight spaced arms, each about $67 \mathrm{~cm}$ long, $10 \mathrm{~cm}$ wide, $20 \mathrm{~cm}$ deep, with a food cup at the end, and all radiating from an octagonal central platform. Before the test, the mice were treated with fasting to maintain their body weight at $80-85 \%$ of their previous weight when eating ad libidum; and throughout the whole test, the diet was controlled to maintain the mice at this weight level. In the adaptation training, $100 \mathrm{mg}$ of food was put in every food cup at the end of every arm and the mice were placed on the central platform, allowing them to travel freely to each arm for food. After 10 minutes or all the food in eight food cups was eaten up, the adaptation training was ended. This training lasted for three days, two times a day. In the formal test, food was put into 4 cups randomly and the test was ended after 10 minutes or all the food was checked for. The test lasted for 7 days with one time per day. The test time, the frequency of the mice entering into each arm, bristling their hair and standing were all recorded. The failure to visit the arm containing food was considered as spatial reference memory error (long-term memory test), which reflected the memory formation of the mice during long-term memory test. Re-entry into the arms would result in a spatial working memory error (short-term memory test), which reflected the temporary memory storage of the mice in a single test. Action capability of the mice should be taken as an inclusion criterion and those bristling or standing for too many times should be excluded.

\subsection{Western Blot to Detect the Expression of IL-1ß and BDNF in Hippocampuses}

$20 \%$ urethane $(1.6 \mathrm{~g} / \mathrm{kg})$ was injected into the abdominal cavity of the mice for anesthesia and their brains were harvested to isolate hippocampuses which were immediately put into liquid nitrogen for freezing. Then the SDS lysate was added into the liquid nitrogen followed by protein phosphatase inhibitor for tissue catalase with ultrasound and low temperature centrifugation, after which the supernatant was extracted and stored at $-80^{\circ} \mathrm{C}$. $12 \%$ SDS-PAGE gel was adopted for electrophoretic separation of the supernatant and then the protein on the gel was transferred onto the PVDF membrane which was then sealed with 5\% skim milk at room temperature for 1 hour and incubated with primary antibody at $4^{\circ} \mathrm{C}$ overnight. Then the protein on the PVDF membrane was rinsed with PBS for 3 times and every time for 10 minutes, and then incubated with horseradish peroxidase-labeled secondary antibody (1: 5000, Abcam) for 1 hour. After another rinse with PBS for 3 times and every time for 10 minutes it was stained with ECL and exposed. Image-pro method was employed to analyze the total gray value of each band which was finally standardized by $\beta$-actin total gray value. The primary antibodies used in this experiment include rabbit anti-mouse BDNF antibody (1: 800, Cell Signaling Technology), rabbit anti-mouse IL-1 $\beta$ antibody (1: 500, Abcam), mouse antimouse $\beta$-actin antibody (1: 1000, Abcam). 


\subsection{Statistical Method}

The data are shown with mean \pm standard error $(x \pm S E M)$. ANOVA was employed for data analysis of different groups followed by Dunnett's test for evaluation. The Western blot results were tested with Two-way ANOVA. $\mathrm{P}<0.05$ indicates that the difference is statistically significant.

\section{A $W T / I L-1 R K O$}
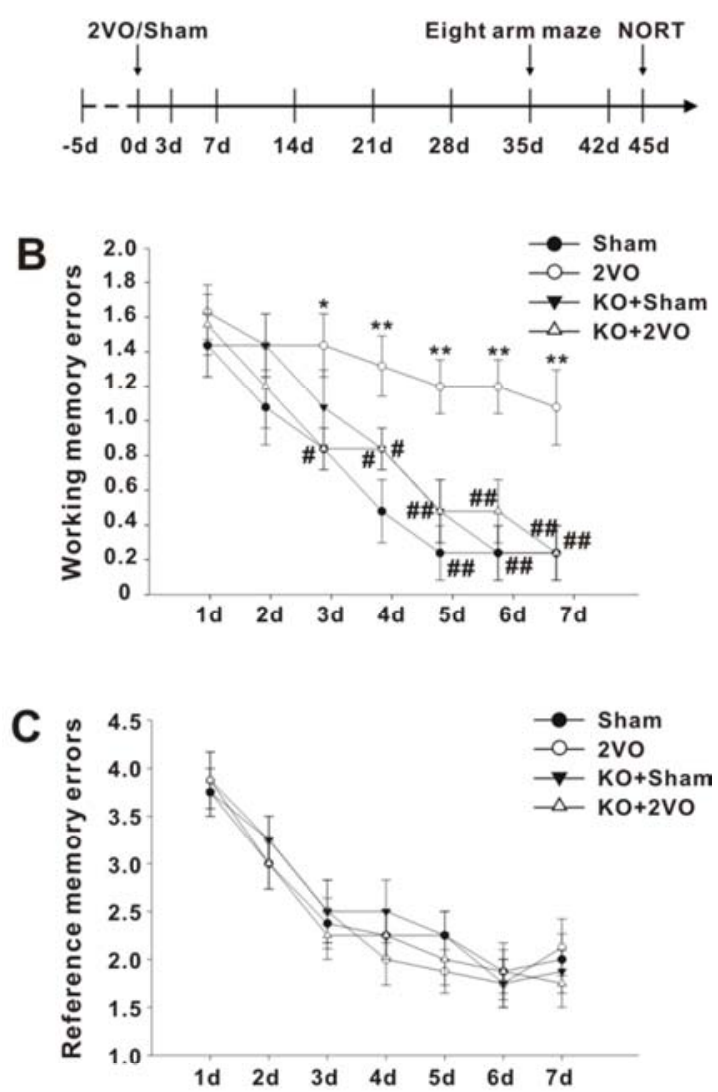

D

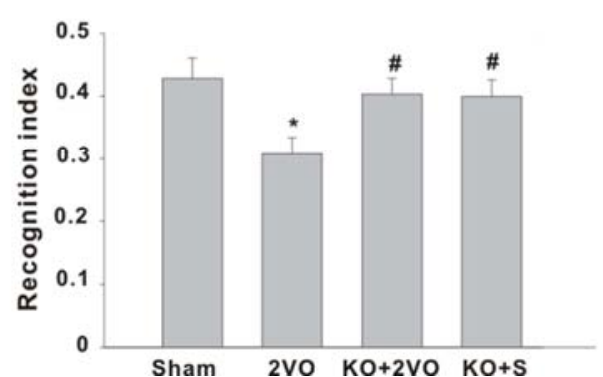

Figure 1. Changes of memory in wild type and IL-1R knock-out mice after bilateral common carotid artery ligation.

A. Experimental flow chart: 5 days of adaptation in feeding cage, $2 \mathrm{VO}$ and Sham surgery on day 0 , eight arm radial maze test on the $36^{\text {th }}-42^{\text {th }}$ day following surgery, novel object recognition test on the $43^{\text {th }}-45^{\text {th }}$ day following surgery; B. The average number of working memory errors in sham group, $\mathrm{KO}-2 \mathrm{VO}$ group and $\mathrm{KO}$-sham group decreases overtime while that in $2 \mathrm{VO}$ group declines very slightly; $\mathrm{C}$. There is no significant difference in average number of cognitive reference memory errors among four groups; D. Recognition index of $2 \mathrm{VO}$ group declines compared with that of sham group while recognition index of $\mathrm{KO}-2 \mathrm{VO}$ group increases in comparison with that of $2 \mathrm{VO}$ group. Compared with sham group, $* \mathrm{P}<0.05$, $* * \mathrm{P}<0.01$; compared with 2 VO group, ${ }^{\#} \mathrm{P}<0.05,{ }^{\# \#} \mathrm{P}<0.01$

\section{Results}

\subsection{Eight-Arm Radial Maze Test Showed that IL-1R Knockout Significantly Improved 2 VO-Induced Working Memory Impairment, But Did Not Affect the Reference Memory}

Working memory and reference memory of the mice in four groups were tested with eight-arm radial maze test on the 36th-42th day. The results showed that there was significant difference in the decline in average number of working memory errors between $2 \mathrm{VO}$ group and sham group $(\mathrm{P}<0.05$, Fig.1B). The decline in $2 \mathrm{VO}$ group was not obvious while in sham group, the decline was more obvious than that in $2 \mathrm{VO}$ group during the $3 \mathrm{rd}-7$ th day, which began on the second day and became significant on the third day. This result showed that working memory impairment exacerbated obviously during the 40th-42th day after bilateral common carotid artery ligation. Average number of working memory errors in $\mathrm{KO}-2 \mathrm{VO}$ group declined slightly compared with those in $2 \mathrm{VO}$ group and there was significant difference in the decline between two groups during the 3rd-7th day, which indicated that IL-1R knockout could improve greatly the 2VO-induced working memory impairment. There was no marked decline in the average number of working memory errors in both $\mathrm{KO}$-sham group and $\mathrm{KO}-2 \mathrm{VO}$ group compared with that in sham group but the difference was not significant $(\mathrm{P}>0.05$, Fig.1B). And there was no significant difference in the average number of reference memory errors among the four groups $(\mathrm{P}<0.05$, Fig. $1 \mathrm{C})$, which indicated that neither sham operation nor $2 \mathrm{VO}$ operation could affect animal's reference memory.

\subsection{Novel Object Recognition Test Showed That IL-1R Knockout Significantly Improved 2 VO-Induced Short-Term Memory Impairment}

In this study, the old and novel object recognition test was adopted to test the short-term memory of mice. The results showed that there was significant difference in the recognition index between sham group and $2 \mathrm{VO}$ group ( $\mathrm{P}<0.05$, Fig.1D). Recognition index of $2 \mathrm{VO}$ group decreased dramatically compared with that of sham group. This result indicated that short-term memory impairment manifests on the 45th day following bilateral common carotid artery ligation, which was consistent with the results of eight-arm radial maze test. Compared with that of the $2 \mathrm{VO}$ group, the recognition index of KO-2VO group increased $(\mathrm{P}<0.05)$, indicating that IL-1R knockout could significantly improve the short-term memory disorder caused by $2 \mathrm{VO}$. There was no significant difference in the recognition index between sham group and $\mathrm{KO}$ groups (KO-sham group and $\mathrm{KO}-2 \mathrm{VO}$ group $)(\mathrm{P}>0.05$, Fig. 1D).

\subsection{Western Blot to Detect the Expression of IL-1ß and BDNF in Hippocampuses}

To investigate the role of IL-1R knockout in improving the 2VO-induced working memory disorder, we used Western Blot to detect the expression level of IL-1 $\beta$ and BDNF in 
hippocampuses of each group. The results showed that the level of IL-1 $\beta$ in $2 \mathrm{VO}$ group was much higher than that in sham group ( $\mathrm{P}<0.05$, Fig. $2 \mathrm{~A}, \mathrm{~B})$ while the level of $\mathrm{BDNF}$ was much lower $(\mathrm{P}<0.05$, Fig. $2 \mathrm{~A}, \mathrm{C})$. The level of IL-1 $\beta$ in the KO-2VO group declined $(\mathrm{P}<0.05$, Figure $2 \mathrm{~A}, \mathrm{~B})$ and the level of BDNF increased ( $\mathrm{P}<0.05$, Figure $2 \mathrm{~A}, \mathrm{C}$ ) compared with those in $2 \mathrm{VO}$ group. There was no significant difference in the level of IL-1 $\beta$ and BDNF between KO-sham group and sham group ( $\mathrm{P}>0.05$, Fig. 2, A, B, C). These results indicated that IL-1R knockout could inhibit the increase of IL-1 $\beta$ caused by $2 \mathrm{VO}$, which resulted in an inhibition of the decline in BDNF.

A

Sham 2Vo KO+2Vo KO+Sham

IL-1 $\beta$

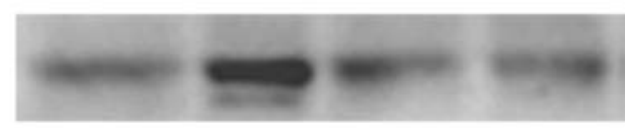

BDNF

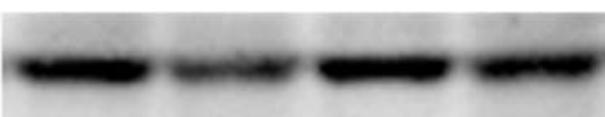

$\beta$-actin

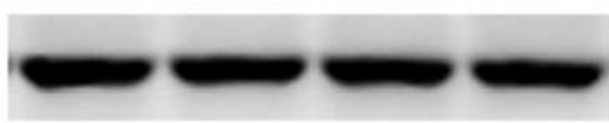

B

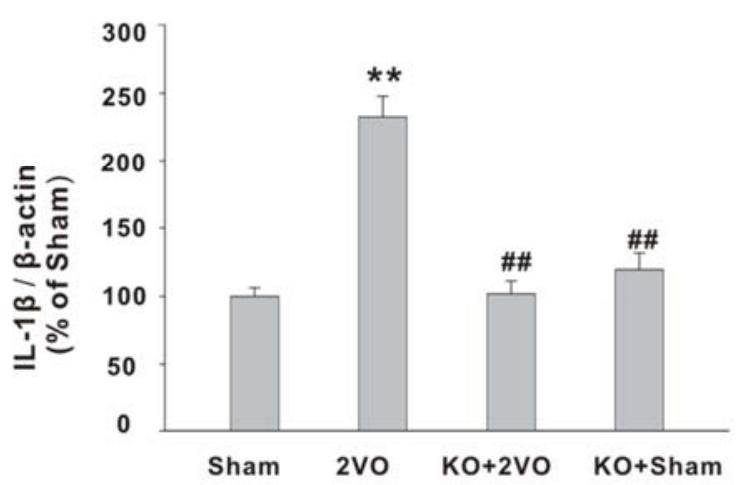

C

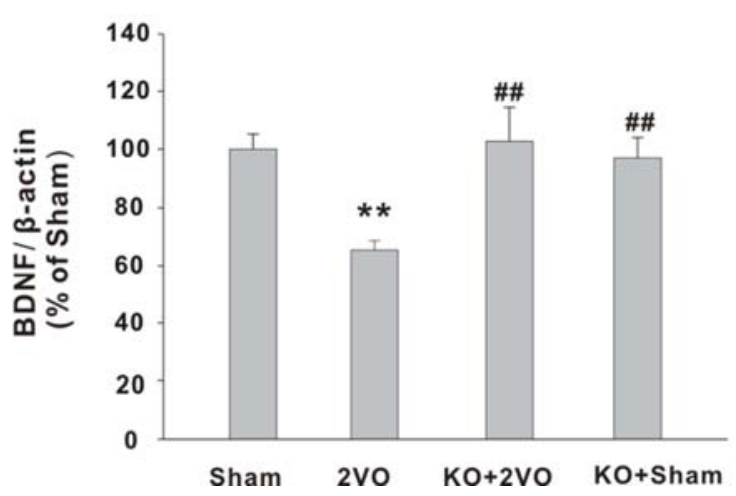

Figure 2. Expressions of $I L-1 \beta$ and BDNF in hippocampus of wild type mice and IL-1R knock-out mice after bilateral common carotid artery ligation.

A. Western Blot is used to detect the levels of IL-1 $\beta$ and BDNF in each group; B. The expression of IL-1 $\beta$ significantly increases in wild type mice after 2VO surgery and IL-1R knockout can inhibit 2VO-induced overexpression of IL-1 $\beta$; C. The level of BDNF in $2 \mathrm{VO}$ group decreased dramatically, and IL-1R knockout relieves the decrease of BDNF expression induced by $2 \mathrm{VO}$; Compared with sham group, ${ }^{*} \mathrm{P}<0.05$, ${ }^{*} * \mathrm{P}<0.01$; compared with $2 \mathrm{VO}$ group, ${ }^{\#} \mathrm{P}<0.05,{ }^{\# \#} \mathrm{P}<0.01$.

\section{Discussion}

Chronic cerebral ischemia-induced memory impairment has raised more and more public concerns. Chronic cerebral ischemia is a common brain injury condition, in which the brain is subjected to low perfusion, resulting in impaired brain function. This injury process involves various complicated pathological changes including cortical atrophy, leukoaraiosis, degeneration of cortical and hippocampal neurons, cortical and hippocampal neuronal degeneration and gliocyte proliferation, etc. [8-9]. Besides, a series of damage mechanisms such as glial cell activation, increased or decreased neurotransmitters, activation of oxidative stress, and neuro immunization which all play a critical role in the development of Bins-wanger disease, Alzheimer's disease (AD), Vascular dementia (VD) and so on, are involved under chronic cerebral ischemia [10-11]. Microglia of neuroprotective and neurotoxic properties are now generally considered as immunological cells widely distributed in the central nervous system. Microglia normally acts as the first and main form of active immune defense in the central nervous system, cleaning up waste but produce neurotoxicity when over activated. Some researchers believe that under chronic cerebral ischemia, microglia activities increase and over-activation makes them release a variety of cytokines and other cytotoxins, ultimately resulting in brain damage of varying degrees [12]. In the central nervous system, IL-1 $\beta$ mainly, synthesized and secreted mainly by microglia, is one of the important cytokines. Normally, the level of IL-1ßis relatively low, but when trauma, inflammation or ischemia rapidly activates microglia, the IL-1 $\beta$ is often released in large quantities. And excessive IL-1 $\beta$ can change neuronal synaptic plasticity and reconstruct neural network [13-14], eventually resulting in brain injury of varying degrees. Brain-derived neurotrophic factor (BDNF), one of the most neurotrophic factors found in the body, is mainly expressed in the central nervous system and reaches peak level in the cortex and hippocampus. BDNF mainly plays its role by combining with TrkB (tyrosine kinase B). A large number of studies have found that when developing chronic cerebral ischemia, the level of BDNF decreases, leading to a reduction in the supply of nutrition factors to brain and thus to the change and death of brain cells, which are often considered closely associated with cognitive disorders [15-17].

In this study, behavioral analysis was performed on wild type mice and IL-1R knock-out mice by eight-arm radial maze test and novel object recognition test to investigate whether working memory after bilateral common carotid artery ligation will change and whether it will be improved in IL-1R knock-out mice after the same operation. The study showed that the average number of working memory errors in wild type mice declined slightly after bilateral common carotid artery ligation but was still higher than that in sham group ( $(\mathrm{P}$ $<0.05$ ), which indicated that the working memory capacity of wild type mice declined dramatically. However, there was no significant difference in the average number of reference memory errors between $2 \mathrm{VO}$ group and sham group, which 
indicated that the reference memory ability of wild type mice was not affected after $2 \mathrm{VO}$. In addition, the level of IL- $1 \beta$ in hippocampus in $2 \mathrm{VO}$ group was significantly higher than that of Sham group $(\mathrm{P}<0.05)$ while the level of BDNF declined enormously compared with that of sham group $(\mathrm{P}<0.05)$. To analyze the correlation between the levels of IL-1 $\beta$ and BDNF and its effect on the working memory ability, we performed $2 \mathrm{VO}$ operation on IL-1R knock-out mice. We found that the level of IL- $1 \beta$ decreased dramatically while the level of BDNF increased greatly compared with those of $2 \mathrm{VO}$ group (wild type mice) $(\mathrm{P}<0.05)$. Besides, the average working memory error in $\mathrm{KO}-2 \mathrm{VO}$ group was significantly improved compared with that in $2 \mathrm{VO}$ group $(\mathrm{P}<0.05)$, and there was no significant difference between KO-2VO group and sham group $(\mathrm{P}>0.05)$.

These data fully show that in the chronic cerebral ischemia mouse models, produced by bilateral common carotid artery ligation, overexpression of IL-1 $\beta$ in hippocampus causes decreased expression of BDNF, which is closely associated with working memory impairment of mice. IL-1 $\beta$ and BDNF in hippocampuses of the IL-1R knock-out mice return to normal levels after $2 \mathrm{VO}$ followed by improved working memory of the mice, which proves that decrease in BDNF caused by increased IL-1 $\beta$ in hippocampus is one of the possible mechanisms leading to working memory capacity impairment of mice. Bilateral common carotid artery ligation is currently considered as a good way to produce chronic cerebral ischemia model [7] because the hippocampus is very sensitive to ischemia and bilateral common carotid artery ligation usually leads to memory impairment in mice. This study provides a new insight into the pathogenesis of chronic cerebral ischemia-induced memory impairment and thus a new scientific basis and possible therapeutic regimen for the prevention of memory impairment and improvement of memory when developing chronic cerebral ischemia.

\section{Conclusions}

Increase in the level of the IL- $1 \beta$ in hippocampus after bilateral common carotid artery ligation can lead to decrease in the level of the BDNF, which may cause memory impairment. This study provides a new insight into the pathogenesis of chronic cerebral ischemia-induced memory impairment and thus a new scientific basis and possible therapeutic regimen for the prevention of memory impairment and improvement of memory when developing chronic cerebral ischemia.

\section{References}

[1] Sivakumar L, Camicioli R, Butcher K. Factors associated with cognitive decline in transient ischemic attack patients. Can J Neurol Sci, 2014, 41(3):303-13.

[2] Amantea D, Nappi G, Bernardi G, Bagetta G, Corasaniti MT.
Post-ischemic brain damage: pathophysiology and role of inflammatory mediators. Febs Journal, 2009, 276(1):13-26.

[3] Zhou Y, Zhang J, Wang L, Chen Y, Wan Y, He Y, et al. Interleukin-1 $\beta$ impedes oligodendrocyte progenitor cell recruitment and white matter repair following chronic cerebral hypoperfusion. Brain Behav Immun, 2017, 60:93-105.

[4] Leal G, Afonso PM, Salazar IL, Duarte. Regulation of hippocampal synaptic plasticity by BDNF. Brain Research, 2014, 1621:82-101.

[5] Chen G, FU Q, Cao J, Mi W. Effect of propofol on brain-derived neurotrophic factor and tyrosine kinase receptor $\mathrm{B}$ in the hippocampus of aged rats with chronic cerebral ischemia. Neural Regen Res, 2012,7(21):1645-9.

[6] Tanila H. The role of BDNF in Alzheimer's disease. Neurobiol Dis, 2017,97(Pt B):114-118.

[7] Toyama K, Koibuchi N, Uekawa K, Hasegawa Y, Kataoka K, Katayama T, et al. Apoptosis signal regulating kinase 1 is a novel target molecule for cognitive impairment induced by chronic cerebral hypoperfusion. Arterioscler Tthromb Vvasc Biol, 2014,34( 3): 616-625.

[8] Wang M, Norman JE, Srinivasan VJ, Rutledge JC. Metabolic, inflammatory, and microvascular determinants of white matter disease and cognitive decline. Am J Neurodegener Dis, 2016, 5(5):171-177.

[9] Brassai A, Suvanjeiev RG, Bán EG, Lakatos M. Role of synaptic and nonsynaptic glutamate receptors in ischaemia induced neurotoxicity. Brain Res Bull, 2015, 112:1-6.

[10] Shukla V, Shakya AK, Perez-Pinzon MA, Dave KR. Cerebral ischemic damage in diabetes: an inflammatory perspective. J Neuroinflammation, 2017,14(1):21.

[11] Alawieh A, Elvington A, Tomlinson S. Complement in the Homeostatic and Ischemic Brain. Front Immunol, 2015,6:417.

[12] Xiong XY, Liu L, Yang QW. Functions and mechanisms of microglia/macrophages in neuroinflammation and neurogenesis after stroke. Prog Neurobiol, 2016, 142:23-44.

[13] Udeochu JC, Shea JM, Villeda SA. Microglia communication: Parallels between aging and Alzheimer's disease. Clin Exp Neuro immunol, 2016, 7(2):114-125.

[14] Ransohoff RM. How neuroinflammation contributes to neurodegeneration. Science, 2016, 353(6301):777-83.

[15] Mizui T, Ishikawa $\mathrm{Y}$, Kumanogoh $\mathrm{H}$, Kojima M. Neurobiological actions by three distinct subtypes of brain-derived neurotrophic factor: Multi-ligand model of growth factor signaling. Pharmacol Res, 2016, 105:93-8.

[16] Zhang ZH, Wu LN, Song JG, Li WQ. Correlations between cognitive impairment and brain? derived neurotrophic factor expression in the hippocampus of post-stroke depression rats. Mol Med Rep, 2012, 6(4):889-93.

[17] Patterson SL. Immune dysregulation and cognitive vulnerability in the aging brain: Interactions of microglia, IL-1 $\beta$, BDNF and synaptic plasticity. Neuropharmacology, 2015, 96(Pt A):11-8. 